\title{
Effective short-range Coulomb correction to model the aggregation behavior of ionic surfactants
}

DOI:

$10.1063 / 1.4954063$

Document Version

Accepted author manuscript

Link to publication record in Manchester Research Explorer

\section{Citation for published version (APA):}

Burgos-Mármol, J. J., Solans, C., \& Patti, A. (2016). Effective short-range Coulomb correction to model the aggregation behavior of ionic surfactants. Journal of Chemical Physics, 144. https://doi.org/10.1063/1.4954063

\section{Published in:}

Journal of Chemical Physics

\section{Citing this paper}

Please note that where the full-text provided on Manchester Research Explorer is the Author Accepted Manuscript or Proof version this may differ from the final Published version. If citing, it is advised that you check and use the publisher's definitive version.

\section{General rights}

Copyright and moral rights for the publications made accessible in the Research Explorer are retained by the authors and/or other copyright owners and it is a condition of accessing publications that users recognise and abide by the legal requirements associated with these rights.

\section{Takedown policy}

If you believe that this document breaches copyright please refer to the University of Manchester's Takedown Procedures [http://man.ac.uk/04Y6Bo] or contact uml.scholarlycommunications@manchester.ac.uk providing relevant details, so we can investigate your claim.

\section{OPEN ACCESS}




\title{
Effective short-range Coulomb correction to model the aggregation behavior of ionic surfactants
}

\author{
J. Javier Burgos-Mármol, ${ }^{1, *}$ Conxita Solans, ${ }^{2}{ }^{\dagger}$ and Alessandro Patti ${ }^{1, \ddagger}$ \\ ${ }^{1}$ School of Chemical Engineering and Analytical Science, \\ The University of Manchester, The Mill. Sackville Street, M13 9PL, Manchester, UK \\ ${ }^{2}$ Institut de Química Avançada de Catalunya (IQAC-CSIC) and CIBER de Bioingeniería, \\ Biomateriales y Nanomedicina (CIBER-BBN), C/ Jordi Girona, 18-26 - 08034 Barcelona, Spain
}

\begin{abstract}
We present a short-range correction to the Coulomb potential to investigate the aggregation of amphiphilic molecules in aqueous solutions. The proposed modification allows to quantitatively reproduce the distribution of counterions above the critical micelle concentration (CMC) or, equivalently, the degree of ionization, $\alpha$, of the micellar clusters. In particular, our theoretical framework has been applied to unveil the behavior of the cationic surfactant $\mathrm{C}_{24} \mathrm{H}_{49} \mathrm{~N}_{2} \mathrm{O}_{2}^{+} \mathrm{CH}_{3} \mathrm{SO}_{4}^{-}$, which offers a wide range of applications in the thriving and growing personal care market. A reliable and unambiguous estimation of $\alpha$ is essential to correctly understand many crucial features of the micellar solutions, such as their viscoelastic behavior and transport properties, in order to provide sound formulations for the above mentioned personal care solutions. We have validated our theory by performing extensive lattice Monte Carlo simulations, which show an excellent agreement with experimental observations. More specifically, our coarse-grained model is able to reproduce and predict the complex morphology of the micelles observed at equilibrium. Additionally, our simulation results disclose the existence of a transition from a monodisperse to a bidisperse size distribution of aggregates, unveiling the intriguing existence of a second CMC.
\end{abstract}

\section{INTRODUCTION}

Surfactants are amphiphilic molecules made up by a hydrophilic head and a hydrophobic tail. In aqueous solutions, at the so-called critical micelle concentration (CMC), surfactants form aggregates, commonly known as micelles. The structural features of these clusters, such as size, shape and packing, are deeply related to some key physico-chemical parameters (temperature, $p H$, salt concentration, etc.) [1-8] as well as to the molecular architecture of their building blocks [9]. Their spontaneous aggregation or self-assembly, being rather recurrent in soft matter systems, is driven by a complex competition between enthalpic and entropic forces, which might be dramatically altered by almost intangible thermal fluctuations of few $k_{B} T$, with $T$ the absolute temperature and $k_{B} \simeq 1.38 \times 10^{-23} \mathrm{~J} \mathrm{~K}^{-1}$ the Boltzmann's constant.

As generally accepted, the formation of micelles has mostly an entropic origin, with the hydrophobic effect playing a crucial role $[10,11]$. More specifically, hydrocarbon chains cannot form hydrogen bonds with water molecules, and, as a consequence, surfactants in aqueous solutions tend to stay at the interface separating water from another liquid (an oily phase, for instance) or a gas (air). Nevertheless, isolated surfactants do exist in the bulk and determine the reorientation of the water molecules around their hydrocarbon tail. This rearrangement minimizes the disruption of the hydrogen bonds and creates a sort of water cage (or shell) solvating the surfactant tails. However, due to this broken symmetry, the mobility of the water molecules forming the solvation shell results to be substantially reduced as well as their configurational entropy $[9,12,13]$. To counterbal- ance this condition, which becomes thermodynamically unfavorable at increasing surfactant concentration, free surfactant molecules aggregate into micelles.

Due to its practical impact in several key areas of industrial interest, including personal care and household cleaning products, micellization is a widely investigated phenomenon and its mechanism is generally wellunderstood. Nevertheless, specific intermolecular interactions and the balance between their entropic and enthalpic nature, being at the heart of the self-assembly process, are still under debate, especially when ionic surfactants are involved. While experimental techniques, such as tensiometry and light scattering, are very powerful to determine the phase and aggregation behavior of micellar systems, molecular simulation can provide an insight into the molecular details, contribute to shed light on the forces driving the self-assembly, and thus optimize the formulation of targeted products.

Fully atomistic models have been applied to investigate phase equilibrium and dynamics of amphiphilic systems, such as their adsorption on specific interfaces [14, 15]. However, these models cannot efficiently supply a comprehensive picture of the self-assembly process because of the relevant time scales, being in the order of microseconds [16]. Coarse-grained (CG) models, where groups of atoms are assembled into single interaction sites, are able to explore larger time scales by drastically reducing the number of degrees of freedom involved. While this representation lacks the full chemical details of real amphiphilic molecules, it still retains the relevant physical parameters driving their micellization. On the basis of these considerations, self-assembling amphiphilic systems have been generally investigated by lattice CG models, 
which provide a discretized rather than continuous representation of the phase space, as in the theory of polymer solutions by Flory and Huggins [17]. Despite the remarkable scientific interest on the phase and aggregation behavior of non-ionic surfactants over the last three decades [18-25], much less attention has been devoted to ionic surfactants, most probably because of the additional complexity arising from the presence of charged molecules. As a consequence, significant effort was made to improve the efficiency of existing models and hence address the fundamental issues of micellization of ionic surfactants. In this context, Bhattacharya et al. applied an effective Debye length in a Debye-Hückel potential to describe the self-assembly of ionic surfactants by offlattice Monte Carlo (MC) simulations in two dimensions. Their model however does not explicitly include counterions and cannot provide an insight into their distribution in solution [26]. Cheong and Panagiotopoulos developed a lattice model to predict the micellization properties of Sodium Dodecyl Sulfate (SDS) [27]. While their model explicitly include counterions, the simulation results underestimate the experimental CMC, micelle size, and degree of ionization, most probably because of a lack of short-range head-head and head-tail repulsions.

More recently, LeBard et al. applied CG Molecular Dynamic simulations to investigate the aggregation behavior of several ionic surfactants, including SDS [28]. In their model, originally proposed by Shinoda, De Vane and Klein, [29, 30], these authors explicitly included the effect of a water-like solvent made up by beads representing, each of them, three water molecules. They observed an interesting transition from spherical to elongated micelles as a function of the hydrocarbon chain length. Additionally, the work by Shinoda and coworkers highlights the importance of properly treating the long-range nature of the Coulomb force even when CG models are employed. This conclusion is also supported by our simulation results. Although most of the models on ionic surfactants assume a point unit charge at the headgroup or counterion, quantum chemical methods unveiled that the charge can partially distribute to the rest of the molecule, more significantly on the $\alpha$-methylene group and less on the remaining alkyl chain [31]. This charge distribution has an important effect on surfactant self-assembly and can explain the polarity of micelle cores. Models incorporating multiple beads have been employed to describe the charge distribution in water [32] and ionic molecules [33].

In this work, we propose a lattice model including a short-range correction to the Coulomb interaction that is able to accurately reproduce the experimental degree of ionization, which determines the extent of counterion condensation (effective charge) on the micelles and thus their formation and mutual interaction $[34,35]$. This correction, justified by theoretical considerations involving the chemical architecture of the surfactant, depends on the distance between charges and is calculated only at the beginning of the simulation, thus reducing the implementation process to a fast table lookup algorithm. Our theoretical model is validated experimentally by investigating the micellization of Ricinoleamidopropyltrimonium methosulfate $\left(\mathrm{C}_{24} \mathrm{H}_{49} \mathrm{~N}_{2} \mathrm{O}_{2}^{+} \mathrm{CH}_{3} \mathrm{SO}_{4}^{-}\right)$, a monovalent cationic surfactant with a long hydrophobic chain and a bulky counterion, commercialized as Varisoft RTM 50. Parametrization of the model was carried out on the basis of the experimental $\mathrm{CMC}$ at $25^{\circ} \mathrm{C}$, that was used to set the short-range tail-tail interaction strength. This represents a substantial difference from the model proposed in Ref. [27], where the model parameters were obtained from experimental data not related tappendix.styo micellization. Beside the fundamental challenges associated to its complex counterion, this surfactant is of relevant interest among formulation industries with core business in personal care products (conditioning shampoos and foams) or cosmetics (skin cleaners). To the best of our knowledge, this work represents the first attempt to describe its aggregation behavior.

This article is structured as follows. In Section II, we describe the experiments performed to generate the data used for the parametrization of our model. The theoretical aspects of the model and simulation methodology are exposed in Section III, where we discuss the correction to the Coulomb potential and justify its application. In Section IV, the simulation results and their comparison with experiments are presented. Finally, some conclusions wrap up the paper.

\section{EXPERIMENTAL METHODOLOGY}

Surface tension. A common method to determine the $\mathrm{CMC}$ is to measure the surface tension of the aqueous solution containing the surfactant. As more surfactant accumulates at the water-air interface, the surface tension of water decreases and, finally, achieves a stable plateau once the amount of surfactant in solution has reached its saturation value. This phenomenon occurs smoothly, with the surface tension decreasing linearly with the logarithm of the concentration. The intersection between the two straight lines describing the transition and saturation regimes, determines the concentration at which micelles form, that is the CMC [36]. To measure the surface tension, we employed a tensiometer Krüss processor $\mathrm{K} 12$ at a temperature $T_{l a b}=25^{\circ} \mathrm{C}$ and analyzed samples at different surfactant concentrations around the CMC. We applied the Wilhelmy method, which consists of a platinum plate of known dimensions connected to a balance, dipped into the solution and raised back slowly until the weight is maximum. After equilibration, the force obtained and the plate's dimensions allow to calculate the surface tension [37]. Since the commercial product is a dilution of approximately $40 \%$ of surfactant in water, 
we used thermo-gravimetrical analysis to determine the exact percentage in mass of amphiphile.

Conductivity. The experimental value of the degree of ionization has been determined by measuring the change in slope of the electrical conductivity, $\kappa$, versus the total surfactant concentration, $c_{\text {surf }}$, across the CMC. It is known that $\kappa$ increases linearly with $c_{\text {surf }}$ in aqueous solutions and displays an abrupt change at the CMC [9]. Our measurements were carried out for different concentrations with a conductimeter Crison GLP 31 at $T_{l a b}$. Above the CMC, the positively charged surfactant heads, mostly concentrating at the micellar corona, attract the negatively charged counterions. This electrostatic attraction is stronger than the thermal energy $k_{B} T$ and drives the counterions to condense on the micellar aggregates [13]. In particular, the degree of ionization, $\alpha$, which is the fraction of counterions that are dissociated from the micelles and thus leave them charged, can be calculated as the ratio between the slopes of $\kappa$ versus $c_{\text {surf }}$ above and below the $\mathrm{CMC}[9]$ :

$$
\alpha=\frac{m_{>}}{m_{<}}
$$

where $m_{>}$and $m_{<}$represent the slope $d \kappa / d c_{\text {surf }}$ above and below the CMC, respectively.

\section{MODEL AND SIMULATIONS}

\section{III.1. Model}

Our CG model is a modification of the lattice model originally proposed by Larson to study the micellization of non-ionic block copolymers [18], and, more recently, different lyotropic liquid crystals, including lamellar, hexagonal, and cubic mesophases [38-40]. In particular, space is organized into a three-dimensional cubic network of sites which are occupied by the solvent, the ionic surfactant molecules or their counterion. The model surfactant, $T_{5} H^{+} C^{-}$, is made up of $n_{T}=5$ beads constituting the hydrophobic tail, $n_{H}=1$ bead which represents the hydrophilic head carrying a positive unit charge, and $n_{C}=1$ negatively charged counterion modeled as a single bead. An equal number of positive and negative charges keeps the whole system electrically neutral. A schematic representation of our CG ionic surfactant is depicted on Fig. 1. Solvent molecules, which occupy the available lattice sites left empty by the surfactant chains, are implicitly included [18]. As a general constraint, no overlaps between beads are allowed.

While in Larson's model all the beads have the same size (the lattice unit length), the diameter of the $H^{+}, T$, and $C^{-}$beads in our model is set according to the average bond lengths and Van der Waals radii of the original amphiphilic molecule. The length of the hydrophobic

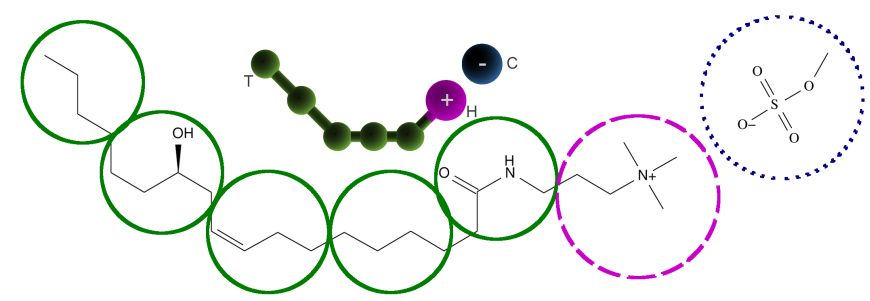

FIG. 1. Schematic representation of the CG ionic surfactant $T_{5} H^{+} C^{-}$and corresponding chemical structure. Green, magenta, and blue beads represent the hydrophobic tail $(T)$, hydrophilic head $\left(H^{+}\right)$, and the counterions $\left(C^{-}\right)$, respectively. Junctions between beads represent bond lengths, which change with orientation. Solid, dashed, and dotted circles over the chemical structure are a guide for the eye representing $T, H^{+}$, and $C^{-}$beads, respectively.

backbone, made up by $18 \mathrm{C}-\mathrm{C}, 2 \mathrm{C}-\mathrm{N}$, and $1 \mathrm{C}=\mathrm{C}$ bonds, is $L_{T}=29.5 \AA$, including the Van der Waals radius of the methyl group, while the angle formed by three consecutive carbon bonds is $\theta_{C C C}=109.5^{\circ}$. By coarse-graining the tail in $n_{T}=5$ beads of identical size, their resulting diameter is $\sigma_{T}=L_{T} / n_{T} \approx 5.9 \AA$, which we take as our model's unit length. By contrast, the diameter of the hydrophilic head $\left(\sigma_{H}\right)$ and counterion $\left(\sigma_{C}\right)$ are approximately the same and equal to $9.5 \AA$. To embody this difference in the geometrical arrangement of a lattice model, surfactant heads and counterions are represented by larger beads with diameter $\sigma_{H}=\sigma_{C}=\sqrt{2} \sigma_{T}$. Although the original surfactant tail is not a mere saturated hydrocarbon chain, the five $T$ beads are here modeled identically, with no attempt to incorporate their chemical details into our CG model. While this choice makes our model less realistic, it provides the unquestionable benefit of reducing its complexity by keeping the primary ingredients promoting self-assembly: $(i)$ the tendency of the hydrocarbon chains to cluster together and (ii) the solubility of the hydrophilic heads. The lattice coordination number, set to $z=26$, implies the presence of fluctuating bond lengths, which only assume values limited by the lattice geometry: $\sigma_{T}, \sqrt{2} \sigma_{T}$, or $\sqrt{3} \sigma_{T}$. In particular, $R_{z}=\sqrt{3} \sigma_{T}$ is the cut-off of the short-range $T-T$ interactions. It should be noticed that, since the size of $C$ and $H$ beads exceeds the lattice unit length, their neighboring sites at distance $\sigma_{T}$ can only be occupied by the implicit solvent. This constraint determines the condensation of counterions and limits the chain's mobility around the micellar corona.

In addition to these excluded volume interactions, our force field comprises the long-range electrostatic interaction between charges, $U_{i j}^{0}$ and the effective short-range interaction between tail groups, $\epsilon_{T T}$. The former is described by the Coulomb's law:

$$
U_{i j}^{0}=\frac{e^{2}}{4 \pi \varepsilon_{r} \varepsilon_{0}} \frac{z_{i} z_{j}}{r_{i j}}
$$


where $z_{H}=1$ and $z_{C}=-1$ are the charges carried by $H^{+}$and $C^{-}$beads, respectively, in units of the elementary charge $e, r_{i j}$ the distance between two charges, $\varepsilon_{r}=78.54$ the relative permittivity (dielectric constant) of water at $T=298.15 \mathrm{~K}$, and $\varepsilon_{0}=8.854 \cdot 10^{-12} \mathrm{Fm}^{-1}$ the vacuum permittivity. The short-range interaction, $\epsilon_{T T}$, is only defined for pairs of nearest or diagonallynearest $T$ beads. The remaining short-range bead-bead interactions, $\epsilon_{i j}$, are set to zero. The nature of these interactions can be better understood by considering the Flory-Huggins theory for polymer solutions [17], where the global interaction established between two beads $i$ and $j$ is defined as

$$
\omega_{i j}=\epsilon_{i j}-\frac{1}{2}\left(\epsilon_{i i}+\epsilon_{j j}\right),
$$

and it is related to the Flory-Huggins parameter $\chi_{i j}=$ $z \beta \omega_{i j}$, with $\beta=1 / k_{B} T$. More specifically, $\chi_{i j}$ defines the nature of the enthalpic contribution to the free energy of self-aggregation between species $i$ and $j$ [17]. Regardless the specific value assigned to the short-range effective interaction, Eq. (3) states that contacts between identical beads do not contribute to the energy change, that is $\omega_{i i}=0$. Since all the energy terms for the formation of unlike pair contacts, $\epsilon_{i j}$, are set to zero, the only global interactions contributing to the energy change are those involving at least one $T$ bead. More specifically, $\omega_{T H}=$ $\omega_{T C}=\omega_{T S}=-\epsilon_{T T} / 2$, whereas $\omega_{H S}=\omega_{C S}=\omega_{H C}=$ 0 . The value of $\epsilon_{T T}$ is set according to the procedure described in section III.3.

\section{III.2. A short-range effective correction to the Coulomb interaction}

The above described model is a rather naïve representation of an aqueous solution containing an ionic surfactant. It neglects at least two major ingredients that can dramatically influence the counterions' condensation on the micelles: $(i)$ the asymmetric distribution of the electric charges in the counterions, and (ii) the low polarizability of the micellar core. To incorporate both effects into our model, we propose a short-range correction to the electrostatic potential. With regard to point $(i)$, a more realistic picture should consider the rearrangement of the charge according to the local molecular distribution. More specifically, close to a positively charged head, the counterion's electronic cloud, on average equally distributed among the three equivalent oxygen atoms, should orient towards the surfactant head, minimize the distance from its positive charge, and thus increase the strength of their Coulomb interaction. For similar reasons, the negative charges of two neighboring $C^{-}$beads would maximize the distance from each other and thus weaken their Coulomb interaction. As far as point $(i i)$ is concerned, assuming a homogeneous dielectric constant, $\varepsilon$, across the micellar solution might not be very satisfactory when estimating the interaction of charges surrounding a micelle and could underestimate the degree of counterions' condensation. The incorporation of both effects leads to a modified Coulomb potential that reads:

$$
U_{i j}^{\delta \varepsilon}=\frac{e^{2}}{4 \pi \varepsilon_{r} \varepsilon_{0}} \frac{z_{i} z_{j}}{G_{\varepsilon}\left(r_{i j}+2 \delta_{i j} F_{\delta}\right)},
$$

where $\delta_{i j}$ depends on the nature of the interacting beads, whereas $F_{\delta}=F_{\delta}\left(r_{i j}\right)$ on the distance between them. In particular, $\delta_{i j} / \sigma_{T}=-\sqrt{2} / 2, \sqrt{2}$, or 0 , for the $H^{+}-C^{-}$, $C^{-}-C^{-}$, and $H^{+}-H^{+}$interactions, respectively. The function $F_{\delta}$ reads:

$$
F_{\delta}\left(r_{i j}\right)=\frac{1}{e^{\frac{r_{i j}-R_{\delta}}{\Sigma_{\delta}}}+1}
$$

where $R_{\delta} / \sigma_{T}=\sqrt{2}$ is the minimum distance allowed between two charged beads. More specifically, the highest deviations from the unmodified Coulomb potential emerge at $r_{i j}=R_{\delta}$. The constant $\Sigma_{\delta}=R_{z}-R_{\delta}$ is the width of $F_{\delta}$. The contribution of $F_{\delta}$ to the Coulomb potential is neglected for distances lower than the diameter of a charged bead, that is $r_{i j} / \sigma_{T}<\sqrt{2}$.

The second correction to the Coulomb potential accounts for the spatial inhomogeneity of the dielectric constant in a micellar solution. Due to significant change of the dielectric properties in the vicinity of an interface, the incorporation of a space-dependent dielectric constant is crucial in many different fields, including electrokinetics [41] and solvation of salt ions [42]. Its rigorous description can be provided by applying the Poisson equation. Nevertheless, the calculation of the induced charges at each simulation step makes solving the Poisson equation very computationally demanding, especially for three-dimensional systems. The recent introduction of a variational formulation of the polarization charge density simplified the explicit calculation of the electrostatic potential $[43,44]$. In our model, to incorporate the spatial inhomogeneity of the dielectric constant, we introduce an effective correction, $G_{\varepsilon}=G_{\varepsilon}\left(r_{i j}\right)$, that can be easily tuned on the basis of experimental conductivity measurements. In particular, the effective dielectric constant is defined as follows:

$$
\varepsilon^{\prime}=\varepsilon_{r} \varepsilon_{0} G_{\varepsilon}=\varepsilon_{r} \varepsilon_{0}\left(1-\xi F_{\varepsilon}\right)
$$

where

$$
F_{\varepsilon}\left(r_{i j}\right)=\frac{1}{e^{\frac{r_{i j}-R_{\varepsilon}}{\Sigma_{\varepsilon}}}+1},
$$


where $R_{\varepsilon} / \sigma_{T}=\sqrt{5}$ is the radius of gyration of the micelles, calculated with the unmodified Coulomb potential (Eq. (2)), and $\Sigma_{\varepsilon}=R_{z}-R_{\delta}$. The condensation parameter, $\xi \in[0,1]$, is heuristic and characteristic of our model, and its purpose is to modulate the intensity of the short range correction. Its value is intimately related to the surfactant studied, and is set on the basis of the experimental degree of ionization. By contrast, the functions $F_{\delta}$ and $G_{\epsilon}$, which are proposed from qualitative considerations, are parametrized in agreement with the electrostatic properties and the chemical structure of the molecules involved. Although the validity of the proposed short-range Coulomb correction is tested here for a particular surfactant, our model can be applied to other ionic surfactants in water solutions. More specifically, by tuning the tail-tail interaction strength and the condensation parameter, and eventually incorporating the effect of the charge distribution in the surfactant heads and counterions, we can study the aggregation behavior of long-chain cationic and anionic surfactants, including, for instance, CTAB and SDS, respectively. The interested reader is referred to the supplemental material at [URL] for additional details on the model proposed here.

\section{III.3. Simulation Methodology}

Preliminary simulations were performed to set the parameters of our force field, that is the interaction energy $\epsilon_{T T}$ and the condensation parameter $\xi$. More specifically, we first simulated a system with $N=100$ molecules in the canonical $(N V T)$ ensemble at the same concentration as the experimental CMC and gradually increased the value of the interaction energy, $\epsilon_{T T}$, until micelles were formed. At $T=298.15 \mathrm{~K}$, this was detected at $\epsilon_{T T} / k_{B} T=-0.503$. Although the $N V T$ ensemble was appropriate to accurately set the value of $\epsilon_{T T}$, it is usually rather inefficient for analyzing the aggregation behavior of amphiphiles at concentrations close to the CMC. Therefore, apart from this initial setting, all the remaining simulations were performed in the grand canonical $(\mu V T)$ ensemble, at a chemical potential in the range $-27.8 \leq \mu / k_{B} T \leq-21.2$, with $\mu / k_{B} T \approx-24.5$ the chemical potential at the CMC.

To set the value of the condensation parameter, $\xi$, in Eq. (7), a number of simulations were performed at $\mu / k_{B} T=-24.1$ and $\epsilon_{T T} / k_{B} T=-0.503$. At this state point, which is just above the CMC, we computed the degree of ionization, $\alpha$, for different values of $\xi$ and compared it with its experimental value. The degree of ionization is the ensemble average of chains ionized in a cluster and is calculated as follows:

$$
\alpha=\left\langle 1-\sum_{i=1}^{N_{c l}} \frac{N_{c, i}}{N_{a, i}}\right\rangle,
$$

where $N_{c l}$ is the total number of clusters in solution, $N_{c, i}$ the number of counterions that condensed on cluster $i$, and $N_{a, i}$ the number of chains in the same cluster. In our model, a counterion is considered to be bound to a micelle, if it is within the coordination shell of at least one surfactant head belonging to that micelle. The value of the condensation parameter giving the best approximation of the degree of ionization above the $\mathrm{CMC}$ was found to be $\xi=0.3$ as indicated in Fig. 2.

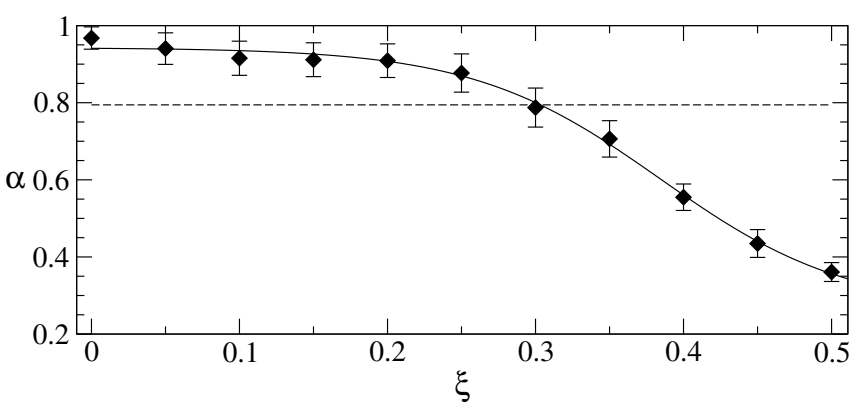

FIG. 2. Micellar degree of ionization, $\alpha$, as a function of the condensation parameter, $\xi$, at $\mu / k_{B} T=-24.1$ and $\epsilon_{T T} / k_{B} T=-0.503$. The dashed line indicates the experimental value of $\alpha$ at the CMC. The solid line is a fitting of the simulation points and intersects the experimental value of $\alpha$ at $\xi=0.3$.

Due to its long-range nature, the electrostatic potential must incorporate the interactions between beads distributed over all the periodic images of the simulation box. To this end, we employed the Ewald summation method, which divides the total interactions into shortrange and long-range contributions, estimated in the real and Fourier space, respectively $[45,46]$. To improve the efficiency of our calculations, the long-range interactions between any pair of sites on the lattice, including their periodic images, were pre-computed at the beginning of each simulation by employing 337 Fourier-space wave vectors and a real-space damping parameter set to $\kappa_{d u m p}=5$. Given the discrete translational symmetry of a lattice, these Fourier-space contributions can be stored in an array and accessed along the simulation via a fast table lookup algorithm [47]. By contrast, our proposed corrections to the Coulomb potential, which arise from the asymmetric distribution of the electric charges in the counterions and the spatial inhomogeneity of the dielectric constant, are computed in the real space. The resulting deviations from the Coulomb potential $U_{i j}^{0}$ are restricted to short distances and have no effect for $r_{i j} / \sigma_{T} \geq 2 \sqrt{2}$, as can be appreciated from Fig. 3 for the particular case of $H^{+}-C^{-}$interactions. Similar results for the $C^{-}-C^{-}$and $H^{+}-H^{+}$interactions are provided as supplemental material [URL].

The initial configurations consisted of surfactant chains sequentially placed in the lattice, which were allowed to 


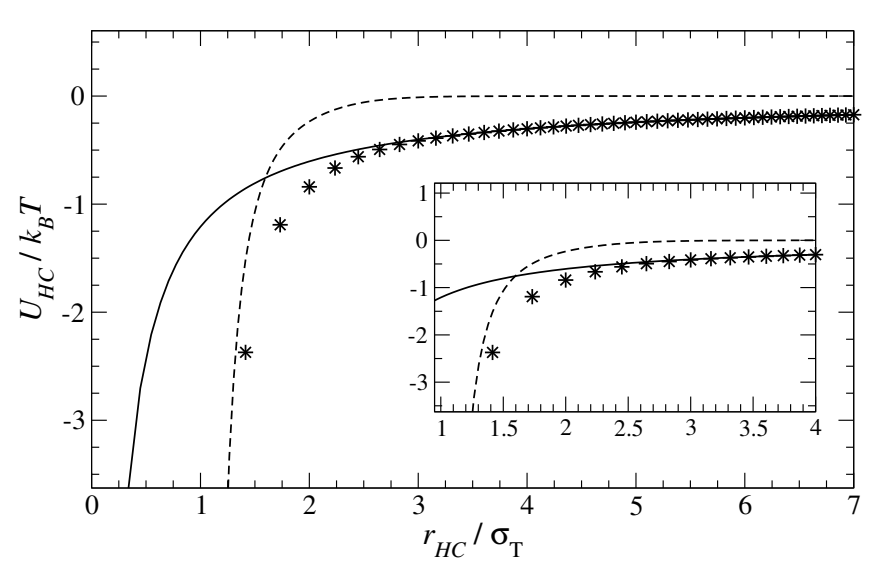

FIG. 3. Coulomb potential, $U_{H C}^{0}$ (solid line), and modified Coulomb potential, $U_{H C}^{\delta \varepsilon}$ (symbols), as a function of the distance between heads and counterions. The dashed line gives the difference between these two potentials, $\Delta U_{H C}^{\delta \varepsilon} \equiv$ $U_{H C}^{\delta \varepsilon}-U_{i j}^{0}$, which is negligible for $r_{H C} / \sigma_{T} \geq 2 \sqrt{2}$. The minimum distance at which the modified potential is applied is $r_{H C} / \sigma_{T}=\sqrt{2}$. The inset displays the behavior of the three functions at short distances.

relax at a very high temperature by temporarily switching off the effect of charges. This created a completely random distribution of the surfactant and the starting configuration for the equilibration run. Surfactant chains $T_{5} H^{+}$have been moved by reptation and configurational bias regrowth $[45,48]$, whereas counterions by random displacements of length $\Delta$, with $0 \leq \Delta \leq L / 2$ in the three directions. A typical mix of attempted MC moves consisted of $50 \%$ reptation, $30 \%$ configurational bias, $10 \%$ counterion displacements, and $10 \%$ of insertion/removal attempts to exchange mass and energy with a reservoir at constant chemical potential and temperature. All kinds of moves were accepted or rejected according to the standard Metropolis algorithm and satisfy the detailed balance [45]. The exchange of $T_{5} H^{+} C^{-}$molecules with the reservoir was performed by following the method proposed by Orkoulas and Panagiotopoulos [49]. Additional details are provided in the supplemental material [URL]. Systems were considered to be at thermodynamic equilibrium with the reservoir if the number of chains achieved a steady value within statistical fluctuations.

To have an insight into the structural properties of the micellar clusters, we calculated their size distribution and degree of elongation. More specifically, the cluster size distribution indicates the preferential size of the micelles and their degree of dispersion in solution. In other words, it is the probability $P\left(N_{g}\right)$ of observing a cluster with aggregation number $N_{g}$ :

$$
P\left(N_{g}\right)=\frac{\left\langle\phi_{N_{g}}\right\rangle V}{\left\langle\sum n_{i}\right\rangle N_{g}\left(n_{H} v_{H}+n_{T} v_{T}\right)}
$$

where $v_{i}$ is the volume occupied by a $T$ or $H^{+}$bead, $\phi_{N_{g}}$ is the volume fraction of clusters with aggregation number $N_{g}, n_{i}$ the amount of clusters with $N_{g}=i$, and $\langle\ldots\rangle$ denotes ensemble average. The degree of elongation of a micelle is strictly related to its three radii of gyration, $R_{g, 1} \leq R_{g, 2} \leq R_{g, 3}$, which are the eigenvalues of the tensor of gyration. These three parameters are here conveniently combined to describe the anisotropy of a micelle in terms of eccentricity and triaxiality. More specifically, the eccentricity is defined as

$$
e^{2}=1-a^{2} / b^{2}
$$

where $a$ and $b$ are the length of the micelle's minor and major semi-axes, respectively. The former is defined as the quadratic mean of $R_{g, 1}$ and $R_{g, 2}$, whereas the latter is set equal to $R_{g, 3}$. The triaxiality allows to distinguish between prolate and triaxial micelles and is defined as

$$
\Delta e^{2}=\Delta a^{2} / b^{2}
$$

where $\Delta a^{2}=\left(R_{g, 2}^{2}-R_{g, 1}^{2}\right) / 2$. Values of the eccentricity and triaxiality for several geometries are shown in Table I.

TABLE I. Limit values of eccentricity, $e^{2}$, triaxiality, $\Delta e^{2}$, and relative weight of the three radii of gyration, $R_{g, i}$, for several micellar geometries.

\begin{tabular}{|c|c|c|c|}
\hline \hline Shape & Radii of gyration ratios & $e^{2}$ & $\Delta e^{2}$ \\
\hline Spherical & $R_{g, 1}^{2} \lesssim R_{g, 2}^{2} \lesssim R_{g, 3}^{2}$ & $\approx 0$ & $\approx 0$ \\
\hline Prolate & $R_{g, 1}^{2} \lesssim R_{g, 2}^{2} \ll R_{g, 3}^{2}$ & $\approx 1$ & $\approx 0$ \\
\hline Oblate & $R_{g, 1}^{2} \ll R_{g, 2}^{2} \lesssim R_{g, 3}^{2}$ & $\approx 1 / 2$ & $\approx 1 / 2$ \\
\hline Triaxial & $R_{g, 1}^{2} \ll R_{g, 2}^{2} \ll R_{g, 3}^{2}$ & $\approx 1$ & $\gtrsim 0$ \\
\hline \hline
\end{tabular}

\section{RESULTS AND DISCUSSION}

In this section, we provide experimental and simulation results of the CMC, degree of ionization, and structural properties of the systems studied. The CMC is the concentration of surfactants above which micelles appear in the system. Below the CMC, most of the amphiphilic chains are isolated and the presence of micelles is practically negligible, although there is, in principle, a small probability of observing clusters of any given size. The $\mathrm{CMC}$ was first determined by the tensiometry measurements shown in Fig. 4. Due to a minor experimental uncertainty in the measurements, the transition regime, with only free surfactant monomers existing in solution, provides a dependence of the surface tension on concentration that can be accurately fitted by two straight lines with a slightly different slope. In order to unambiguously identify the $\mathrm{CMC}$, we set its value at 


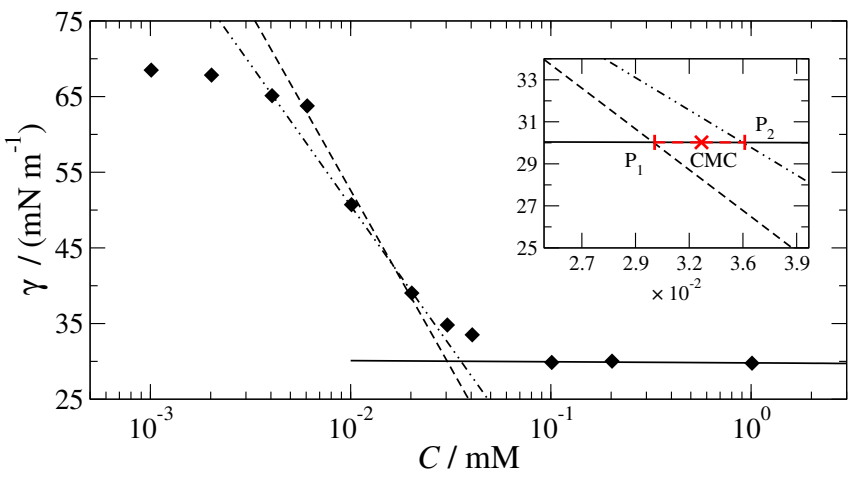

FIG. 4. Surface tension $\gamma$ as a function of surfactant concentration $C$. Dashed and dash-dotted lines are fittings of the experimental data in the transition regime. Experimental data in the saturation regime are fitted by a solid line. In the inset, points $P_{1}$ and $P_{2}$ locate the intersection between the fitting lines of the transition regime and that of the saturation regime. The experimental CMC is considered to be at half distance between $P_{1}$ and $P_{2}$ (red cross).

the midpoint between the intersection points, $P_{1}$ and $P_{2}$, of the straight lines fitting the transition regime with the line fitting the saturation regime. This value, displayed in the inset of Fig. 4 , is $(3.3 \pm 0.3) \times 10^{-2} \mathrm{mM}$, being very similar to the CMC of other cationic surfactants with analogous architecture $[9,50]$. To better quantify the agreement between experimental and simulation results, hereafter all the concentrations will be normalized by the experimental CMC, defining $C^{*} \equiv C / C M C$, where $C M C=3.3 \times 10^{-2} \mathrm{mM}$. The surface tension measurements allow to estimate other relevant physico-chemical parameters of $\mathrm{C}_{24} \mathrm{H}_{49} \mathrm{~N}_{2} \mathrm{O}_{2}^{+} \mathrm{CH}_{3} \mathrm{SO}_{4}^{-}$, which, to the best of our knowledge, are here calculated for the first time. The interested reader is referred to the supplemental material [URL] for further details.

The CMC obtained experimentally was used as input parameter in the $N V T$ MC simulations to set the value of $\epsilon_{T T}$. At this stage, we used the Coulomb potential with no corrections, $U_{i j}^{0}$, and found $\epsilon_{T T} / k_{B} T=-0.503$. With this value of the $T-T$ interaction, the CMC was recalculated in the $\mu V T$ ensemble with the modified form of the Coulomb potential, $U_{i j}^{\delta \epsilon}$, given in Eq. (5) with condensation parameter $\xi=0.3$. By estimating the $\mathrm{CMC}$ in the $\mu V T$ ensemble, our aim was to determine the effect of the modified Coulomb potential on the aggregation behavior of our model surfactant. To this end, the concentration of free chains $\left(C_{\text {free }}^{*}\right)$ and that of chains in aggregates $\left(C_{a g g}^{*}\right)$ were plotted as a function of the total surfactant concentration, $C^{*}$, as shown in Fig. 5 .

At the CMC, the slope $d C_{f r e e} / d C^{*}$ decreases from 1 to approximately 0 , whereas $d C_{a g g} / d C^{*}$ increases from 0 to approximately 1 . This indicates that above the CMC any further addition of surfactant to the solution contributes to increase the concentration of aggregates, but leaves

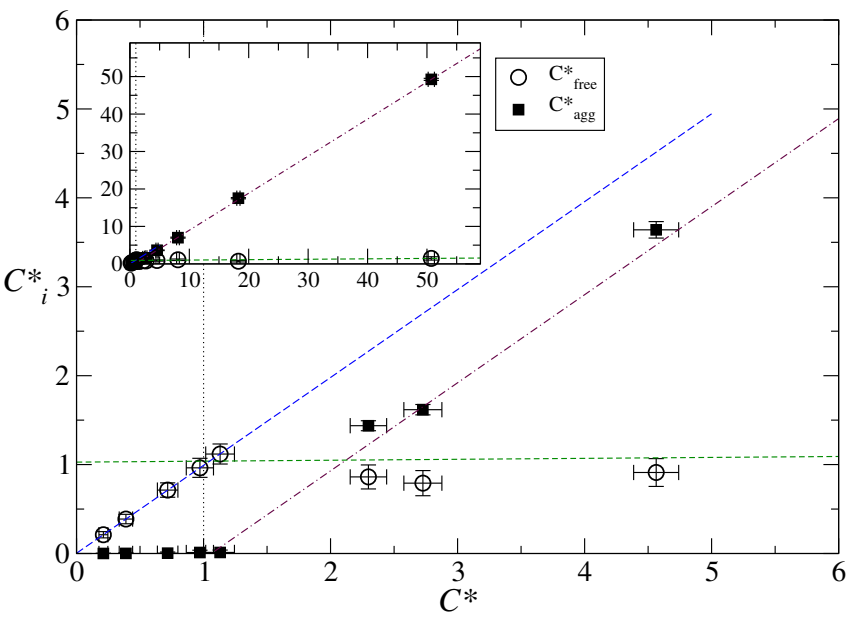

FIG. 5. Concentration of free surfactant $\left(C_{\text {free }}^{*}\right.$, empty circles) and concentration of surfactant in aggregates $\left(C_{a g g}^{*}\right.$, solid squares) versus the total surfactant concentration $\left(C^{*}\right)$ as calculated in the $\mu V T$ ensemble with the modified Coulomb potential and condensation parameter $\xi=0.3$. Dashed lines are guides for the eye. The change of the slope $d C_{f r e e} / d C^{*}$ or $d C_{a g g} / d C^{*}$ determines the CMC, which is indicated by a vertical dotted line. The inset shows larger concentrations.

unchanged that of free molecules. We assume that the concentration at which the change of slope $d C_{\text {free }} / d C^{*}$ (or $d C_{a g g} / d C^{*}$ ) is observed is the CMC, as previously proposed by other authors $[24,51]$. Other definitions of the $\mathrm{CMC}$ are also available $[23,52,53]$.

The value of the CMC determined with the modified Coulomb potential does not show any significant difference from the experimental CMC. As a consequence, one can conclude that the charge density distribution in the counterions and the permittivity of the hydrophobic micellar core have a very weak impact on the CMC, which is adequately predicted by the traditional form of the Coulomb potential provided in Eq. (2). In other words, a simple CG model neglecting key physico-chemical details of a micellar solution of ionic surfactants is still able to provide a good estimate of the CMC as long as the effective interactions between tail beads, $\epsilon_{T T}$, is consistently set. Nevertheless, a close analysis of the aggregation behavior of our ionic surfactant reveals that in order to estimate other properties, such as the aggregation number and degree of ionization, a more sophisticated description of the intermolecular interactions is required. In particular, the degree of ionization has been estimated experimentally by measuring the conductivity, $\kappa$, at different normalized surfactant concentrations, $C^{*}$, as presented in Fig. 6.

Interestingly enough, three separate linear regimes, one below and two above the CMC, with $\kappa \propto(3.3,2.6$, 2.3) $C^{*}$, respectively, have been determined. The straight lines fitting the experimental points in Fig. 6 intersect at $C_{1}^{*}=1.01 \pm 0.20$ and $C_{2}^{*}=6.6 \pm 0.9$. The first con- 


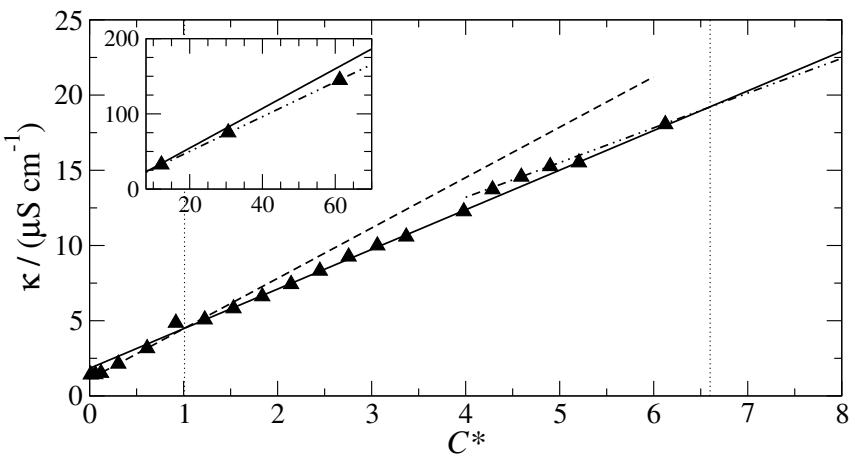

FIG. 6. Experimental conductivity $\kappa$ at several concentrations. Solid triangles represent experimental data. Lines are fits of points below (dashed), slightly above (solid) and well above (dash-dotted) the CMC. The inset contains data of conductivity beyond $C^{*}=8$. Vertical dotted lines locate the intersection of the linear fits of contiguous data sets at $C_{1}^{*}$ (CMC) and $C_{2}^{*}$.

centration, $C_{1}^{*}$, indicates the position of the $\mathrm{CMC}$ and is in excellent agreement with the CMC calculated by surface tension measurements and found by simulations in the $\mu V T$ ensemble. The intersection at $C_{2}^{*}$ would indicate the occurrence of a second $\mathrm{CMC}$, possibly due to a shape transition from spherical to prolate micelles with increasing aggregation number [54]. Sphere-to-rod transitions are not unusual in micellar systems of cationic surfactants and are associated to the dehydration of the micellar interface and simultaneous increase of the interfacial counterion's concentration [55]. In our systems, the degree of ionization, $\alpha$, calculated by conductivity measurements according to Eq. (1), shows an abrupt change at $C^{*}=C_{2}^{*}$, as indicated in Fig. 7 . In particular, $\alpha \approx 0.8$ between the first and second CMC, whereas $\alpha \approx 0.7$ for $C^{*}>C_{2}^{*}$. These experimental results (dashed lines in Fig. 7) have been compared with the simulation results obtained by applying the bare form of the Coulomb potential (empty symbols in the same figure) and the modified form of the Coulomb potential with $\xi=0.3$ (solid symbols).

While the traditional form of the Coulomb potential cannot reproduce the abrupt change of $\alpha$, the simulation results based on our theoretical approach are in very good agreement with the experiments and confirm the existence of a third regime above $C_{2}^{*}$. In this regime, $\alpha$ appears to be substantially reduced as a result of a larger condensation of counterions around the micellar aggregates. Although our simulations cannot be conclusive on the abrupt change of $\alpha$ observed experimentally at $C^{*}=C_{2}^{*}$, they clearly indicate that it decreases significantly as compared to the region of concentrations in between the two CMCs, a tendency not observed when Eq. (2) is applied. Therefore, the local distribution of charges in the $\mathrm{H}^{+}$and $\mathrm{C}^{-}$beads and the spatial heterogeneity of the dielectric constant play an important role in assessing

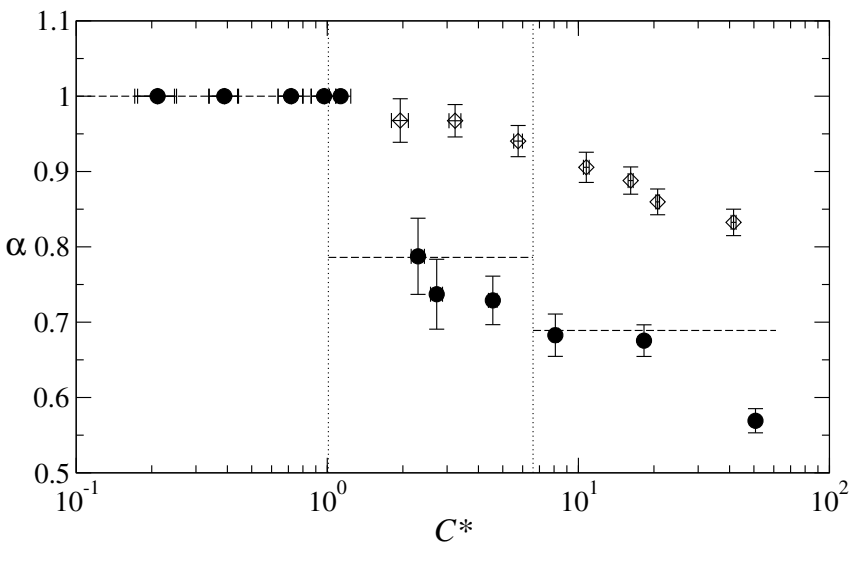

FIG. 7. Degree of ionization as a function of reduced surfactant concentration. Horizontal dashed lines refer to experimental values obtained from conductivity experiments. Symbols refer to simulation results obtained by employing the traditional Coulomb potential (empty diamonds) and the modified Coulomb potential with $\xi=0.3$ (solid circles). Vertical dotted lines indicate the value of the two CMCs and define the borders of the three relevant concentration's intervals.

the condensation of counterions around the micelles and are crucial to reproduce the experimental observations. These results are confirmed by the analysis of the density profiles, $\rho_{i}(r)$, which describe the spatial distribution of a given bead $i$ in concentric spherical shells around the center of the micelles [39]. In particular, they provide relevant details on the counterions' condensation that cannot be gained from the degree of ionization. Density profiles calculated at $\mu / k_{B} T=-24.2$, corresponding to a surfactant concentration in between $C_{1}^{*}$ and $C_{2}^{*}$, are shown in Fig. 8 for systems whose electrostatic interactions are described by Eq. (2) (top frame) and Eq. (4) (bottom frame). The density of counterions on the micelles' surface is larger when the modified Coulomb potential is employed, in good agreement with the results shown in Fig. 7. Regardless the Coulomb potential applied, heads and counterions share the same volume surrounding the micellar core, which is almost completely composed by hydrocarbon chains.

In order to unambiguously ascertain the origin of the second CMC, we estimated shape and size of the micelles observed in solution across the complete range of concentrations studied and up to $C^{*} \approx 50$. In particular, Fig. 9 presents the cluster size distribution (CSD) at concentrations $C_{1}^{*}<C^{*}<C_{2}^{*}$ (top frame) and $C^{*}>C_{2}^{*}$ (bottom frame) for both the traditional and modified form of the Coulomb potential (dashed and solid line, respectively). The narrow CSDs in the top frame, both calculated at $C^{*}=2\left(\mu / k_{B} T=-24.2\right)$, indicate the presence of an essentially monodisperse system of micelles with aggregation number $N_{g} \approx 35$ for the traditional Coulomb potential and $N_{g} \approx 48$ for the modified Coulomb potential. The shifting of the CSD towards larger aggre- 

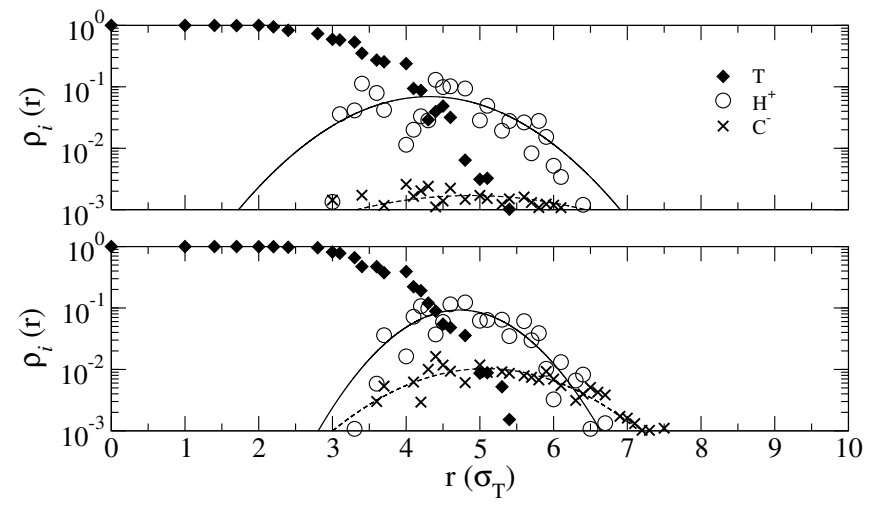

FIG. 8. Density of $T$ (diamonds), $H^{+}$(circles) and $C^{-}$ (crosses) beads as a function of the radial distance from the center of micelles at $C_{1}^{*}<C^{*}<C_{2}^{*}\left(\mu / k_{B} T=-24.2\right)$. The top and bottom frames show density profiles in systems whose Coulomb interactions are described, respectively, by Eq. (2) and Eq. (4). Solid and dashed lines are guides for the eye.

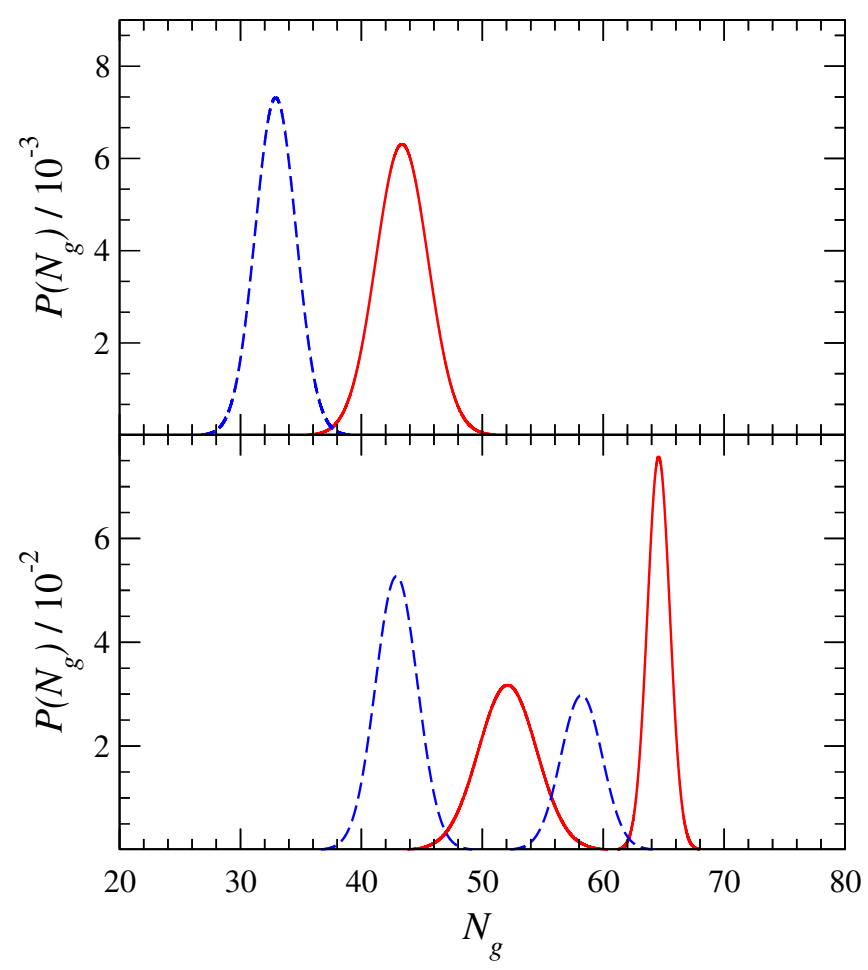

FIG. 9. Cluster size distributions as a function of the aggregation number as obtained by molecular simulation with the traditional (blue dashed lines) and modified (red solid lines) Coulomb potential. The top frame shows monodisperse distributions at $\mu / k_{B} T=-24.2$ and $C^{*} \approx 2$, while the bottom frame shows bidisperse distributions at $\mu / k_{B} T=-21.2$ and $C^{*} \approx 50$.

gation numbers is most probably due to the effect on the short-range interactions between charged beads introduced in the modified Coulomb potential $U_{i j}^{\delta \epsilon}$. More specifically, while $U_{i j}^{\delta \epsilon}$ has no effect on the long-range interactions between charged beads, it produces short- range interactions that are significantly more attractive than those resulting from the traditional Coulomb potential $U_{i j}^{0}$, as Fig. 3 shows. Such stronger attractions promote the efficient packing of the charged beads in the micellar corona by partially compensating the steric effects produced by the lattice geometry. The resulting effect is a larger number of surfactant chains fitting within a single micellar aggregate. For similar reasons, above the second $\mathrm{CMC}$, the micelles' aggregation number increases when the modified Coulomb potential is applied, as confirmed by the bottom frame of Fig. 9, where results are given for systems at $\mu / k_{B} T=-21.2$. However, regardless the electrostatic potential employed, the CSD's profiles are substantially different from those observed at $C_{1}^{*}<C^{*}<C_{2}^{*}$ as two well distinct peaks can be appreciated. These peaks reveal the presence of a bidisperse distribution of micelles with aggregation number $N_{g} \approx 43$ and $N_{g} \approx 58$ for $U_{i j}^{0}$ (dashed curves in Fig. 9 ), and $N_{g} \approx 52$ and $N_{g} \approx 65$ for $U_{i j}^{\delta \epsilon}$ (solid curves in Fig. 9). Bidisperse systems with similar CSDs (not shown here) have been also observed at other concentrations above $C_{2}^{*}$. The peaks of these distribution profiles, indicating the most likely aggregation numbers, keep essentially the same value in the range of concentrations between $C_{1}^{*}$ and $C_{2}^{*}$. In particular, Fig. 10 shows the aggregation number of a system described by the modified Coulomb potential as a function of the total surfactant concentration. A family of micelles consisting of roughly 50 surfactant chains is observed above $C_{1}^{*}$, while a second family of micelles with $N_{g} \approx 60$ appears only above $C_{2}^{*}$. An estimate of their radius, $r_{m i c}$, can be obtained from $r_{m i c}=\sqrt{5 / 3} R_{g}[56]$, with $18 \AA<R_{g}<24 \AA$. Therefore, $r_{m i c}$ vary from $23 \AA$ to $31 \AA$, and the micelles' diameter, which also includes the thickness of the corona (approximately $\sqrt{3} \sigma_{T}=10.2 \AA$ ), varies between $67 \AA$ and $82 \AA$. These values are in good agreement with experimental observations on micellar aggregates made of similar cationic surfactants $[57,58]$. The larger aggregates enhance the condensation of the counterions dispersed in solution and would explain the abrupt decrease of $\alpha$ observed experimentally at concentrations above the second CMC. Despite the $\sim 20 \%$ difference in aggregation number between the two families of micelles in the bidisperse region, the difference between their radii $R$, with $R \propto N_{g}^{1 / 2}$, would be too small to be unambiguously distinguished by experimental techniques, such as Dynamic Light Scattering (DLS). Therefore, while our experimental observations indicate that some sort of change took place at $C_{2}^{*}$, it is only by molecular simulation that we were able to understand its origin and relate it to a structural transition.

With this insight into the dependence of the aggregates' size and aggregation number on the surfactant concentration, we now complete our analysis on the morphology of the micelles by determining their shape and 


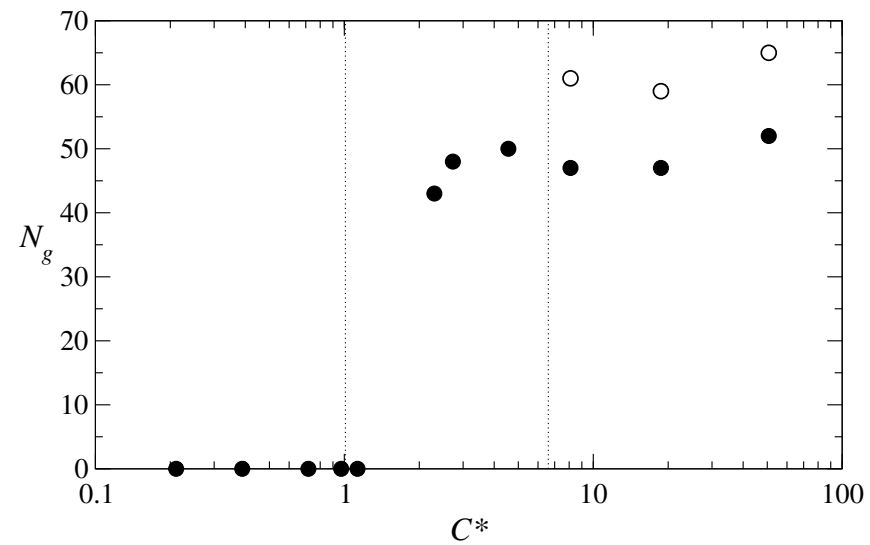

FIG. 10. Aggregation number, $N_{g}$, as a function of the total surfactant concentration, $C^{*}$, as obtained by molecular simulation with the modified Coulomb potential, $U_{i j}^{\delta \epsilon}$. Empty and solid circles at $C^{*}>C_{2}^{*} \approx 6.6$ indicate the presence of bidisperse micelles. Error bars are of the size of the symbols or smaller. Dotted lines represent the values of the two experimental CMCs and delimit the three regimes studied in this work.
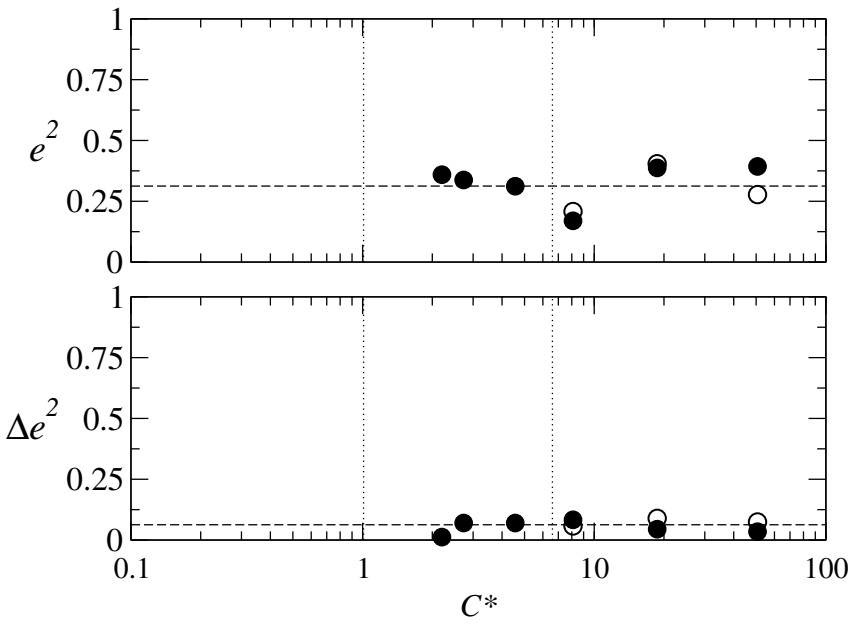

FIG. 11. Eccentricity (top frame) and triaxiality (bottom frame) of aggregates observed in systems described by the modified Coulomb potential. Empty and solid circles at $C^{*}>$ $C_{2}^{*} \approx 6.6$ indicate the presence of bidisperse micelles. Error bars are of the size of the symbols or smaller. Vertical dotted lines represent the values of the two experimental CMCs and delimit the three regimes studied in this work. Horizontal dashed lines denote the average value of eccentricity (top) and triaxiality (bottom).

investigating the eventual occurrence of a shape transition across the second CMC. To this end, the eccentricity, $e^{2}$, and triaxiality, $\Delta e^{2}$, of the aggregates in solution have been calculated as described in Eqs. (10) and (11). The results are shown in Fig. 11 for the modified Coulomb potential $U_{i j}^{\delta \epsilon}$. Both parameters clearly indicate that the morphology of the micellar aggregates has a very weak dependence on the surfactant concentration and does not display any relevant change across the second CMC. In particular, we found that $e^{2}<0.5$ and $\Delta e^{2}<0.1$. According to Table I, we can conclude that the micelles observed in the monodisperse and bidisperse regions are roughly spherical and no shape transition occurs. Switching off the modifications to the electrostatic potential does not alter this picture and maintain both $e^{2}$ and $\Delta e^{2}$ essentially at the same values as those in Fig. 11. As an additional proof supporting this conclusion, we also calculated the intermicellar distance $(I D)$, which was shown to be strongly related to the occurrence of a sphere-to-rod transition [58]:

$$
I D=d_{m i c}\left(\frac{\pi}{6 \phi}\right)^{1 / 3}
$$

where $d_{m i c}$ and $\phi$ are the diameter and volume fraction of the micelles, respectively. In particular, a shape transition is expected when $I D$ is of the order of the size of the micelles [59]. For monodisperse micelles with $d_{m i c}=80 \AA$, the $I D$ in the most concentrated systems studied here is $I D \approx 420 \AA$, which is roughly 5 times the size of the micelles. According to this result, the second CMC determined from conductivity experiments does not correspond to a sphere-to-rod transition.

In the light of these results, we can state that, since our CG model neglects the chemical details of the systems studied, but it is still able to predict the micelles' size transition and the change of the degree of ionization, the second CMC is most probably the mere consequence of the ionic nature and geometry of the surfactant. The cis-trans isomerism of the surfactant due to the double bond between the $12^{t h}$ and $13^{t h}$ carbon atoms of the hydrocarbon tail, completely disregarded in our model, might not be the origin of two separate CMCs, as for instance observed in the micellization of a proline surfactant forming two conformational isomers [60].

\section{CONCLUSIONS}

In summary, we presented a theoretical model incorporating a short-range correction to the Coulomb potential to describe the self-assembly of ionic surfactant molecules in dilute aqueous solutions. In order to evaluate the effects of this correction, we investigated the micellization behavior of Ricinoleamidopropyltrimonium methosulfate, $\mathrm{C}_{24} \mathrm{H}_{49} \mathrm{~N}_{2} \mathrm{O}_{2}^{+} \mathrm{CH}_{3} \mathrm{SO}_{4}^{-}$, a cationic surfactant especially employed in formulation cosmetics. The coarsegrained lattice model used in this work is a solventfree representation of an amphiphilic solution, where the model surfactant, $T_{5} H^{+} C^{-}$, consists of five hydrophobic beads $(T)$, one positively charged hydrophilic head $\left(H^{+}\right)$, and one negatively charged counterion $\left(C^{-}\right)$. Although similar models have been used in the past to describe dilute and concentrated amphiphilic solutions, the novelty 
here arises from incorporating a short-range correction to the Coulomb potential, justified by theoretical considerations on the asymmetric distribution of the electric charges in the counterions and the low polarizability of the micellar core. Incorporating these contributions has an effect on the short-range interactions between charged beads, but does not alter their long-range interaction as compared to the traditional Coulomb potential.

Surface tension and conductivity measurements were performed to obtain the most suitable interaction parameters for the force field used in the model. In particular, the value of the effective attraction between hydrocarbon chains, $\epsilon_{T T}$, was set to reproduce the experimental CMC determined by surface tension. We stress that the value of $\epsilon_{T T}$ was set with no modifications in the Coulomb potential. By contrast, the strength of the electrostatic interaction between charged beads, $\xi$, referred to as condensation parameter, was set to reproduce the counterions' condensation around the micelles, or, equivalently, their degree of ionization, at concentrations close to the CMC. Interestingly enough, conductivity measurements revealed an abrupt decrease of the micelles' degree of ionization at surfactant concentrations well above the CMC. The occurrence of this change, that seems to be related to the presence of a second CMC, was also confirmed by simulations. A detailed computational analysis of the micelles' morphology across the second CMC discarded the occurrence of a sphere-to-rod shape transition, but unveiled a size transition from monodisperse to bidisperse aggregates. In particular, the bidisperse cluster size distribution highlighted the formation of two welldefined families of micelles with pretty different aggregation numbers.

Incorporating a modification to the Coulomb potential does not alter the value of the CMC, most probably because the effect of the charge distribution in the counterions and the permittivity of the micellar core is not particularly significant. Nevertheless, the modified potential determines an increase of the aggregates' size in both the monodisperse and bidisperse solutions. We believe that this is the result of a better packing arrangement of the charged beads, promoted by stronger short-range interactions. Additionally, the modified Coulomb potential is crucial to observe the decrease of the degree of ionization across the two CMCs, which was clearly overestimated by the bare Coulomb potential. Therefore, the spatial heterogeneity of the dielectric constant and the distribution of the charges in the counterions is of fundamental importance to properly address the condensation of counterions in the micellar corona. Although the present coarse-grained model is a rather simplified representation of an ionic surfactant in water, the modifications we have proposed improve significantly its predictability robustness and provide an understanding of the micellization process that cannot fully achieved by experiments.

\section{Acknowledgments}

The authors acknowledge Grants JCI-2010-06943 and CTQ2011-29336-C03 offered by the Spanish Ministry of Science and Innovation, and Grant 2009SGR-961 offered by the Catalan Government. CIBER-BBN is an initiative funded by the VI National R\&D\&i Plan 2008-2011, Iniciativa Ingenio 2010, Consolider Program, CIBER Actions and financed by the Instituto de Salud Carlos III with assistance from the European Regional Development Fund.

* Electronic mail: josejavier.burgos-marmol@manchester.ac.uk

$\dagger$ Electronic mail: csmqci@cid.csic.es

¥ Electronic mail: alessandro.patti@manchester.ac.uk

[1] M. F. Emerson, A. Holtzer, Journal of Physical Chemistry 69 (11), 3718 (1965).

[2] S. Förster, N. Hermsdorf, Macromolecules 35, 4096 (2002).

[3] P. Martínez-Landeira, J. M. Ruso, G. Prieto, F. Sarmiento, J. Chem. Eng. Data 47 (4), 1017 (2002).

[4] M. Benrraou, B. L. Bales, R. Zana, Journal of Physical Chemistry B 69, 13432 (2002).

[5] H. U. Kim, K. H. Lim, Colloids and Surfaces A 235, 121 (2004).

[6] M. Burkhardt, N. Martínez-Castro, S. Tea, M. Drechsler, I. Babin, I. Grishagin, R. Schweins, D. V. Pergushov, M. Gradzielski, A. B. Zezin, A. H. E. Müller, Langmuir 23, 12864 (2007).

[7] P. Palladino, R. Ragone, Langmuir 27, 14065 (2011).

[8] E. B. Zhulina, O. V. Borisov, Macromolecules 45, 4429 (2012).

[9] M. J. Rosen, Surfactants and interfacial phenomena, 3rd ed. (John Wiley \& Sons, Inc. 2004).

[10] P. Müller, Pure and Applied Chemistry 66, 1077 (1994).

[11] D. Chandler, Nature 437, 640 (2005).

[12] C. Tanford, The hydrophobic effect, formation of micelles and biological membranes, 2nd ed. (John Wiley \& Sons, Inc. 1980).

[13] P. C. Hiemenz, R. Rajagopalan, Principles of colloid and surface chemistry, 3rd ed. (Marcel Dekker, Inc. 1997).

[14] H. Domínguez, Journal of Physical Chemistry B 111, 4054 (2007).

[15] W. Zhu, F. S. Romanski, X. Meng, S. Mitra, M. S. Tomassone, European Journal of Pharmaceutical Sciences 42, 452 (2011).

[16] P. Alexandridis, J. F. Holzwarth, T. A. Hatton, Langmuir 9, 2045 (1993).

[17] P. J. Flory, Principles of Polymer Chemistry, (Cornell University Press, Ithaca, NY, 1953).

[18] R. G. Larson, L. E. Scriven, H. T. Davis, Journal of Chemical Physics 83, 2411 (1985).

[19] R. G. Larson, Journal of Chemical Physics 91, 2479 (1989).

[20] A. C. Balazs, C. P. Siemasko, C. W. Lantman, Journal of Chemical Physics 94, 1653 (1991).

[21] A. Z. Panagiotopoulos, M. A. Floriano, S. K. Kumar, Langmuir 18, 1940 (2002). 
[22] Z. A. Al-Anber, J. Bonet Avalos, M. A. Floriano, A. D. Mackie, Journal of Chemical Physics 118, 3816 (2003).

[23] A. Patti, Colloids and Surfaces A 361, 81 (2010).

[24] A. G. Daful, J. Bonet Avalos, A. D. Mackie, Langmuir 28, 3730 (2012).

[25] A. G. Daful, A. D. Mackie, A. Chakrabarti, Journal of Chemical Physics 140104905 (2014).

[26] A. Bhattacharya, S. D. Mahanti, A. Chakrabarti, Journal of Chemical Physics 108, 10281 (1998).

[27] D. W. Cheong, A. Z. Panagiotopoulos, Langmuir 22, 4076 (2006).

[28] D. N. LeBard, B. G. Levine, P. Mertmann, S. A. Barr, A. Jusufi, S. Sanders, M. L. Klein, A. Z. Panagiotopoulos, Soft Matter 8, 2385 (2012).

[29] W. Shinoda, R. DeVane, M. L. Klein, Molecular Simulation 8, 27 (2007).

[30] W. Shinoda, R. DeVane, M. L. Klein, Soft Matter 7, 6178 (2011).

[31] P. Huibers, Langmuir, 15, 7546 (1999)

[32] S. O. Yesylevskyy, L. V. Schäfer, D. Sengupta, S. J. Marrink, PLoS Comput. Biol., 6, e1000810 (2010)

[33] M. V. Fedorov, N. Georgi, A. A. Kornyshev, Electrochemistry Communications, 12, 296 (2010)

[34] A. J. Konop, R. H. Colby, Langmuir 15, 58 (1999).

[35] V. K. Aswal, J. Kohlbrecher, P. S. Goyal, H. Amenitsch, S. Bernstorff, J. Phys.: Condens. Matter 18, 11399 (2006).

[36] K. Holmberg, Handbook of Applied Surface and Colloid Chemistry, Vols. 1862, 1st ed. (John Wiley \& Sons, Inc. 2002).

[37] D. J. Shaw, Introduction to Colloid and Surface Chemistry, 4th ed. (Butterworth-Heinemann, 1992).

[38] F. R. Siperstein, K. E. Gubbins, Langmuir 19, 2049 (2003).

[39] A. Patti, A. D. Mackie, F. R. Siperstein, Langmuir 23, 6771 (2007).

[40] A. Patti, A. D. MacKie, V. Zeleňák, F. R. Siperstein, J. Mater. Chem. 19, (2009).

[41] S. Porada, B. B. Sales, H. V. M. Hamelers, P. M. Biesheuvel, J. Phys. Chem. Lett. 3, 1613 (2012).
[42] Y. Levin, Phys. Rev. Lett. 102, 147803 (2009).

[43] R. Allen, J.-P. Hansen, S. Melchionna, Phys. Chem. Chem. Phys. 3, 4177 (2001).

[44] D. Boda, D. Gillespie, W. Nonner, D. Henderson, B. Eisenberg, Physical Review E 69, 046702 (2004).

[45] D. Frenkel, B. Smit, Understanding Molecular Simulation: From Algorithms to Applications, 2nd ed. (Academic Press, 2002).

[46] M. P. Allen, D. J. Tildesley, Computer Simulations of Liquids (Oxford University, New York, 1987).

[47] A. Z. Panagiotopoulos, S. K. Kumar, Phys. Rev. Lett. 83, 2981 (1999).

[48] M. N. Rosenbluth, A. W. Rosenbluth, J. Chem. Phys. 23, 356 (1955).

[49] G. Orkoulas, A. Z. Panagiotopoulos, J. Chem. Phys. 101, 1452 (1994).

[50] T. F. Tadros, Applied Surfactants. Principles and Applications (Wiley-VCH Verlag GmbH \& Co. KGaA, Weinheim, 2005).

[51] J. Israelachvili, Intermolecular $\&$ Surface Forces, 2nd ed. (Academic Press, 1991).

[52] D. Stauffer, N. Jan, Y. He, R. B. Pandley, D. G. Marangoni, T. Smith-Palmer, J. Chem. Phys. 100, 6934 (1994).

[53] A. D. Mackie, A. Z. Panagiotopoulos, I. Szleifer, Langmuir 13, 5022 (1997).

[54] M. Bergström, Current opinion in colloid \& interface science 22, 46 (2015).

[55] Y. Geng, L. S. Romsted, S. Froehner, D. Zanette, L. J. Magid, I. M. Cuccovia, H. Chaimovich, Langmuir 21, $562(2005)$.

[56] L. A. Feigin, D. I. Svergun, In Structure Analysis by Small-Angle X-Ray and Neutron Scattering (Plenum Press, New York and London, 1987).

[57] J. B. Hayter, J. Penfold, Colloid \& Polymer Sci. 261, 1022 (1983).

[58] H.-U. Kim, K.-H. Lim, Bull. Korean Chem. Soc. 25, No $3(2004)$

[59] J. Yamamoto, H. Tanaka, Nature 409, 321 (2001).

[60] M. Deng, X. Huang, R. Wu, Y. Wang, J. Phys. Chem. B 112, 10509 (2008). 